\title{
Caracterización de un modelo para fomentar la alfabetización matemática en la infancia: vinculando investigación con buenas prácticas
}

\author{
Ángel Alsina, Universidad de Girona (España)
}

Recibido el 10 de octubre de 2016; aceptado el 27 de enero de 2017

Caracterización de un modelo para fomentar la alfabetización matemática en la infancia: vinculando investigación con buenas prácticas

\section{Resumen}

Se presenta un modelo para fomentar la alfabetización matemática en Educación Infantil. El Modelo de Alfabetización Matemática en la Infancia incluye seis fases: matematización del contexto de enseñanza-aprendizaje; conocimientos matemáticos previos de alumnos; aprendizaje de conocimientos matemáticos y documentación en contexto; co-construcción y reconstrucción de conocimiento matemático en el aula; formalización de conocimientos matemáticos adquiridos; y reflexión sistemática sobre la práctica matemática realizada. La caracterización del modelo se fundamenta en los avances de los últimos años en diferentes ámbitos temáticos y agendas de investigación en educación matemática en general y educación matemática infantil en particular. Se consideran contribuciones sobre métodos de enseñanza de las matemáticas y sobre buenas prácticas que provienen principalmente de la Educación Matemática Realista y del Consejo Nacional de Profesores de Matemáticas de Estados Unidos.

Palabras clave. Educación matemática infantil, alfabetización matemática, investigación en educación matemática, buenas prácticas, Educación Matemática Realista.

Caracterização de um modelo para promover a literacia matemática na infância: ligação da investigação com as melhores práticas

\section{Resumo}

Este artigo apresenta um modelo para melhorar literacia matemática na educação infantil. Modelo Literacia Matemática na Infância inclui seis fases: matematização contexto de ensino e aprendizagem; conhecimento matemático anterior de estudantes; aprendizagem conhecimento matemático e documentação no contexto; co-construção e reconstrução do conhecimento matemático na sala de aula; formalização do conhecimento matemático adquirido; e reflexão sistemática sobre a prática matemática. $O$ caracterização é baseada em avanços nos últimos anos em diferentes áreas temáticas $e$ agendas de pesquisa em educação matemática e matemática educação infantil. Especificamente, eles consideram várias contribuições sobre os métodos de ensino de matemática e de boas práticas, principalmente, da Educação Matemática Realista e do Conselho Nacional de Professores de Matemática dos Estados Unidos.

Palavras chave. Educação matemática na infância, literacia matemática, pesquisa em educação matemática, boas práticas, Educação Matemática Realista.

Para citar: Alsina, A. (2017). Caracterización de un modelo para fomentar la alfabetización matemática en la infancia: vinculando investigación con buenas prácticas. Avances de Investigación en Educación Matemática, 12, 59-78.

(C) Sociedad Española de Investigación en Educación Matemática (SEIEM). www.seiem.es 


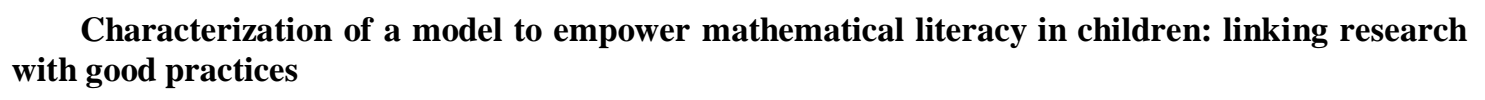

\section{Abstract}

This article presents a model to empower mathematical literacy in Childhood Education. Model of Mathematical Literacy in Childhood includes six phases: mathematisation of the context of teaching and learning; prior mathematical knowledge of students; learning mathematical knowledge and documentation in context; co-construction and reconstruction of mathematical knowledge in the classroom; "Formalization" of mathematical knowledge; and systematic reflection on the mathematical practice done. The characterization of the model draws on advances in recent years in different thematic areas and research agendas in mathematics education in general and Childhood mathematics education in particular. This model considers contributions on methods of teaching mathematics and good practice mainly from the Realistic Mathematics Education and the National Council of Teachers of Mathematics.

Key words. Childhood mathematics education, mathematical literacy, mathematics education research, good practices, Realistic Mathematics Education.

Caractérisation d'un modèle pour promouvoir l'alphabétisation mathématique chez les enfants: liant recherche avec bonnes pratiques

\section{Résumé}

Cet article présente un modèle pour améliorer l'alphabétisation des mathématiques dans l'éducation de la petite enfance. Modèle de l'Alphabétisation Mathématique de la Petite Enfance comprend six phases: mathématisation de le contexte de l'enseignement et l'apprentissage; connaissance mathématique précédente des élèves; apprentissage des connaissances mathématiques et de la documentation dans le contexte; co-construction et reconstruction des connaissances mathématiques dans la salle de classe; "Formalisation" des connaissances mathématiques; et réflexion systématique sur la pratique mathématique. Le caractérisation de modèle est basée sur les progrès de ces dernières années dans différents domaines thématiques et des programmes de recherche dans l'éducation en mathématiques en général et dans l'éducation en mathématiques de l'enfance en particulier. Plus précisément, nous considérons diverses contributions sur les méthodes de l'enseignement des mathématiques et des bonnes pratiques, principalement de l'Education Mathématique Réaliste et le Conseil National des Professeurs de Mathématiques des États-Unis.

Paroles clés. Éducation de la petite enfance, alphabétisation des mathématiques, recherche dans l'éducation en mathématiques, bonnes pratiques, l'Education Mathématique Réaliste.

\section{Introducción}

Durante las últimas décadas la educación matemática infantil ha ido adquiriendo mayor consideración, en gran medida debido a que diversos organismos han expresado sus beneficios para los niños y para la sociedad en general. El Consejo Nacional de Profesores de Matemáticas de Estados Unidos (NCTM, 2003, p. 80) señala que:

Desarrollar una sólida base matemática en los primeros años es especialmente importante para todos los niños. Deben apoyarse sus esfuerzos y su confianza en que aprender matemáticas está a su alcance. Los niños construyen creencias sobre lo que son las matemáticas, sobre lo que significa saber y utilizar matemáticas y sobre sí mismos como aprendices de matemáticas (...). Por consiguiente, es imperativo proporcionarles programas de gran calidad que incluyan matemáticas significativas, y presentarlas de una manera que respete tanto las matemáticas como la naturaleza de los niños.

La Organización para la Cooperación y el Desarrollo Económico señala que está demostrado que los niños que tienen acceso a servicios de educación y cuidados de calidad durante la primera infancia obtienen resultados mucho mejores, equivalentes a un avance de uno o dos años escolares, en pruebas internacionales sobre competencias básicas, como PISA y PIRLS (OECD, 2007). La Asociación Australiana de Profesores 
de Matemáticas y Primera Infancia (2012, p. 2) explica que "todos los niños, en sus primeros años, son capaces de acceder a grandes ideas matemáticas, relevantes para su vida actual y, a su vez, fundamentales para su futuro aprendizaje de las matemáticas y para otros aprendizajes". De Castro, Flecha y Ramírez (2015) y de Castro (2016) indican que estas conclusiones han dado lugar a que varios autores (Clements, 2004; Clements \& Sarama, 2009) e instituciones de referencia internacional, como la Asociación Nacional para la Educación de la Primera Infancia (NAEYC) y el Consejo Nacional de Investigación (NRC), además del NCTM, hayan ido extendiendo su reflexión sobre la educación matemática infantil a edades cada vez menores, llegando a incluir el periodo de 0 a 3 años (Fuson, Clements, \& Beckman, 2009; NAEYC \& NCTM, 2013; NRC, 2014). Otra consecuencia del nuevo panorama es el aumento de las investigaciones sobre educación matemática infantil. En España, estudios bibliométricos de análisis temático de los Simposios de la Sociedad Española de Investigación en Educación Matemática (SEIEM), señalan esta dirección. Mientras que los estudios de Gómez, Cañadas, Bracho, Restrepo y Aristizábal (2011) y de Sierra y Gascón (2001) ponen de manifiesto una escasa productividad de investigaciones sobre educación matemática infantil durante los periodos 1997-2008 y 1997-2010, Alsina (2013) indica que la creación del Grupo de Investigación en Educación Matemática Infantil (IEMI) supone un punto de inflexión que da lugar a un cuerpo de investigaciones más cohesionado. A partir del análisis del contenido matemático, en ese trabajo establezco tres grupos de estudios sobre: 1) la formación inicial de maestros de Educación Infantil, con trabajos desde distintos enfoques como la Teoría de Situaciones Didácticas (TSD), la Teoría Antropológica de lo Didáctico (TAD) o la Educación Matemática Realista (EMR); trabajos basados en métodos de formación activa, como el Aprendizaje Basado en Problemas (ABP) o el Aprendizaje Colaborativo; y trabajos sobre experiencias interdisciplinarias de formación, prácticas externas y trabajos finales de Grado; 2) la adquisición y el desarrollo del pensamiento matemático infantil, con trabajos de análisis de referentes internacionales a nivel curricular (NCTM, 2003) y trabajos que desde un enfoque didáctico se centran en el aprendizaje de contenidos (sobre todo, numeración y cálculo); 3) los recursos y contextos de aprendizaje para favorecer el desarrollo del pensamiento matemático, como contextos de vida cotidiana, juegos, cuentos, etc.

En el contexto europeo también se ha producido una tendencia similar. Edo (2016) analiza la emergencia de la investigación en educación matemática infantil en el grupo Early Years Mathematics (EYM) del Congress of European Research in Mathematics Education (CERME), y constata como desde la creación del grupo en 2009 hasta 2015 ha ido aumentando el número de contribuciones y de investigadores participantes. Esta autora señala que entre los temas que se han tratado en las diferentes ediciones aparece el análisis de las oportunidades de aprendizaje matemático en contextos informales, el papel de los materiales, las diferentes formas de comunicación y representación matemática de los niños pequeños o las evidencias de aprendizaje sobre contenidos específicos, entre otros. El eje común de todos estos trabajos es "el deseo de mejorar el aprendizaje de las matemáticas para los niños pequeños" (Edo, 2016, p. 57).

La finalidad de este artículo es empezar a caracterizar un modelo para empoderar la alfabetización matemática en la infancia, considerando avances en ámbitos temáticos y agendas de investigación en educación matemática infantil durante los últimos años. Para fundamentar dicho modelo se consideran diversas contribuciones sobre los métodos de enseñanza de las matemáticas y sobre buenas prácticas.

\section{Métodos de enseñanza de las matemáticas en Educación Infantil}


En las aulas de Educación Infantil coexisten diversos métodos para enseñar matemáticas. Baroody y Coslick (1998) distinguen cuatro métodos de enseñanza centrados en la actividad matemática del aula, sintetizados por de Castro (2007):

- Enfoque de destrezas: contempla el aprendizaje matemático como la memorización de destrezas básicas a través de la repetición. El objetivo principal es adquirir un conjunto de reglas, fórmulas y procedimientos. Los alumnos son considerados vacíos de contenido e incapaces de comprender por sí mismos la mayor parte de conocimientos matemáticos. Las tareas no acostumbran a estar relacionadas con el entorno cercano y se encuentran alejadas de sus intereses. El énfasis en la repetición hace que los alumnos adquieran destrezas de ejecución aun careciendo de sentido.

- Enfoque conceptual: se empieza a contemplar la necesidad de comprender y adquirir el aprendizaje de procedimientos. Su objetivo principal es conseguir esta adquisición desde la significatividad y la comprensión de los alumnos. Se entiende la enseñanza como un proceso donde es necesario, en ocasiones, hacer uso de dibujos o materiales manipulativos.

- Enfoque de resolución de problemas: se conciben las matemáticas como un espacio en el que los alumnos pueden reflexionar y razonar aquello que les despierta curiosidad. A su vez considera a los niños poseedores de la capacidad de construir sus propios conocimientos. El objetivo principal de este enfoque es introducir a los alumnos en la actividad matemática mediante la resolución de problemas reales y cercanos. El papel del maestro es el de acompañante en el proceso, entendiendo al alumno como protagonista de su aprendizaje.

- Enfoque investigativo: es una combinación entre el enfoque conceptual y el de resolución de problemas. Entiende las matemáticas no solo como adquisición de conceptos y procedimientos sino también como proceso de investigación. Los alumnos deciden el camino que deben recorrer en su aprendizaje matemático y el maestro es su orientador, que actúa solo cuando los alumnos se bloquean ante la tarea. El principal objetivo es conseguir que los alumnos, por sí mismo, lleguen a sus propias conclusiones mediante reflexión, razonamiento, representación, resolución de problemas e investigación.

Considerando los métodos anteriores, López y Alsina (2015) presentan los resultados de una tesis doctoral cuya finalidad es comparar el rendimiento matemático de alumnos de 3 a 6 años y los tipos de andamios que ofrece el profesorado en función del método. Con datos de 149 alumnos y 9 maestras de Educación Infantil, se observan diferencias estadísticamente significativas en el rendimiento matemático de los alumnos que aprenden a través de la metodología de los rincones de trabajo (combinación de los enfoques de resolución de problemas e investigativo), respecto a los que aprenden a través de fichas (enfoque de destrezas) y materiales manipulativos (enfoque conceptual). El análisis cualitativo revela que las ayudas más habituales del profesorado que trabaja a partir de fichas son "suscitar", "instruir" e "informar", en el caso de materiales manipulativos las principales ayudas consisten en "suscitar", "razonar" y "reflexionar" y, en el trabajo a partir de rincones, "razonar", "suscitar" y "orientar". Los resultados, además de poner de manifiesto la heterogeniedad de métodos para enseñar matemáticas en las aulas de Educación Infantil, evidencian que estas diferentes maneras de enseñar tienen efectos diferentes en el rendimiento matemático de los alumnos. Se sugiere que el diseño y la gestión de las prácticas matemáticas acaba determinando el aprendizaje 
matemático de los alumnos. En el siguiente apartado se revisan aportaciones en relación al diseño y la gestión de buenas prácticas matemáticas en las primeras edades.

\section{Buenas prácticas matemáticas en Educación Infantil}

El término "buena práctica" ha sido objeto de debate en la investigación en educación matemática y en el contexto de la educación infantil. En este apartado, se presentan aportaciones, principios o descripciones de buena práctica matemática que se centran en diversos aspectos como el tipo de contenidos, el diseño de actividades, los medios a emplear, la gestión que debería realizarse en el aula o bien la evaluación. Primero se exponen contribuciones genéricas de organismos y autores del ámbito de la educación matemática (NCTM, 2003, 2015; Planas \& Alsina, 2014). Luego se describen aportaciones específicas en el marco de la educación matemática infantil (Asociación Australiana de Profesores de Matemáticas e Infancia, 2012; NAEYC \& NCTM, 2013).

Desde una perspectiva genérica, en el contexto de la definición de los principios y estándares para la educación matemática, NCTM (2003, p. 17) señala que "una enseñanza eficaz requiere conocer lo que los alumnos saben y lo que necesitan aprender; y luego estimularlos y ayudarlos para que lo aprendan bien". Se consideran tres elementos en el marco del "Principio de Enseñanza" de las matemáticas:

- La eficacia docente exige saber matemáticas, tener en cuenta que los alumnos son aprendices y disponer de estrategias pedagógicas. De ahí, el profesorado debería tener diferentes conocimientos (disciplinares, didácticos, etc.)

- Una enseñanza eficaz requiere un entorno en aprendizaje que apoye y estimule. Se trata de plantear propuestas educativas o tareas matemáticas útiles para introducir nociones matemáticas importantes y para retar e implicar intelectualmente a los alumnos. La toma de decisiones respecto al tipo de propuestas debe acompañarse con decisiones sobre la gestión.

- Una enseñanza eficaz requiere tratar continuamente de mejorar. Las buenas prácticas surgen de la observación y reflexión sistemática de la propia práctica.

Los elementos anteriores forman parte de los principios que en la publicación del NCTM de 2003 pretendían describir las características de la educación matemática de calidad. Como puede apreciarse, las principales ideas asociadas al principio de enseñanza se referían a los conocimientos disciplinares y didácticos del profesorado para poder llevar a cabo una buena planificación y gestión de prácticas matemáticas.

Años después se actualizan los principios en el manual De los Principios a la Acción (NCTM, 2015). A diferencia de los principios publicados en 2003, ahora se pretende guiar la educación matemática de los próximos años, los principios se vinculan con prácticas eficaces y se ilustran con ejemplos. En concreto, se describen condiciones, estructuras y políticas que han de darse para que los alumnos aprendan matemáticas, además de abordarse elementos esenciales de la enseñanza y el aprendizaje, del acceso y la equidad, del currículo, o bien otros aspectos como herramientas y tecnología, evaluación y profesionalización. Sugieren acciones que el profesorado debe llevar a cabo para garantizar el éxito de todos en las matemáticas. En concreto, mencionan ocho prácticas basadas en investigaciones que representan un conjunto esencial de habilidades esenciales de enseñanza que se requieren para desarrollar un profundo aprendizaje de las matemáticas: 1) Establecer metas matemáticas basadas en el aprendizaje, 2) Implementar tareas de razonamiento y de resolución de problemas, 3) Usar y vincular las representaciones matemáticas, 4) Favorecer el discurso matemático significativo, 5) 
Plantear preguntas deliberadas, 6) Elaborar la fluidez procedimental a partir de la comprensión conceptual, 7) Favorecer el esfuerzo productivo en el aprendizaje de las matemáticas, 8) Obtener y utilizar evidencias del pensamiento de los estudiantes.

Estas ocho prácticas de enseñanza de las matemáticas son "un conjunto de acciones muy recomendables para todos los docentes, asesores pedagógicos y especialistas en matemáticas, así como para todo el personal administrativo de escuelas y distritos y cada uno de los líderes políticos y responsables de políticas" (NCTM, 2015, p. 4).

Para concretar las características de una buena práctica matemática, Planas y Alsina (2014) retoman los siete principios clásicos de la enseñanza de las matemáticas elaborados por el matemático inglés John Perry y sintetizados en Price (1986, p. 114). A modo de decálogo los completan con tres principios más al final de la lista:

- Tener en cuenta la motivación y los intereses del alumnado.

- Basar lo abstracto en la experiencia concreta para promover la comprensión.

- Emplear actividades que supongan el uso de la mano y el ojo, y no solo de la oreja, en conjunción con el cerebro, así como de los métodos gráficos.

- Adoptar métodos experimentales y heurísticos: experimento, estimación, aproximación, observación, inducción, intuición, sentido común, etc.

- Retrasar el rigor lógico y la preocupación inicial por los fundamentos, y restringir los elementos deductivos formales, admitiendo diversas formas de demostración.

- Simplificar, ensanchar y unificar la materia-disciplina de las matemáticas, e ignorar las divisiones artificiales tradicionales.

- Correlacionar las matemáticas con la ciencia y el trabajo de laboratorio, y relacionar las matemáticas con la vida y sus aplicaciones.

- Recordar la necesidad de incorporar el rigor lógico y la preocupación por los fundamentos en los momentos posteriores a la experiencia concreta.

- Introducir formas de validación de la práctica matemática que no hayan surgido de la implicación del alumnado en las actividades propuestas.

- Generar motivación e interés en el alumnado por problemas matemáticos.

Añaden los tres últimos principios con la intención de cerrar "mejor" el círculo, retomando cuestiones y prácticas matemáticas de importancia que podrían no ser incorporadas en el desarrollo del currículo si solo se tuvieran en cuenta la motivación y los intereses del alumnado o si se retrasara tanto el rigor lógico y la preocupación por los fundamentos que, finalmente, no se volviera a ellos.

Desde la perspectiva de la educación matemática infantil también hay intentos de concreción de lo que se entiende por buena práctica matemática. Se presentan diversas recomendaciones que describen las características que deben tener las prácticas matemáticas en Educación Infantil, tales como el uso de materiales manipulativos, juegos y otros recursos, o bien la incorporación progresiva de lenguaje matemático durante las primeras edades. En este sentido, sigue una síntesis de recomendaciones de la Asociación Australiana de Profesores de Matemáticas e Infancia (2012):

- Atraer la curiosidad natural de los niños para favorecer el desarrollo de las ideas y de la comprensión de las matemáticas infantiles, usando enfoques como el 
juego, el currículo emergente, el currículo centrado en los niños o el currículo iniciado por los niños.

- Asegurar que las ideas matemáticas con las que interactúan los pequeños sean relevantes, usando métodos infantiles de resolución de problemas matemáticos como base para su desarrollo posterior.

- Reconocer que el aprendizaje de las matemáticas es una actividad social que debe ser apoyada y en la que se debe profundizar, tanto a través de la interacción con otros niños, como con los adultos.

- Proporcionar materiales apropiados, espacio, tiempo y otros recursos para animar a todos los niños a implicarse en su aprendizaje matemático. Y reconocer que, aunque los materiales pueden ser importantes en el desarrollo infantil de ideas matemáticas, éstas se desarrollan a través del pensamiento sobre la acción.

- Fijarse en el uso del lenguaje para describir, explicar y justificar ideas matemáticas, reconociendo el importante papel que juega el lenguaje en el desarrollo de todo aprendizaje.

- Evaluar el desarrollo matemático de los niños a través de medios tales como la observación, historias de aprendizaje o debates. Y reconocer que el propósito de dichas evaluación es hacer un seguimiento del desarrollo y facilitar la planificación de las siguientes interacciones, tareas, actividades e intervenciones.

Para la Asociación Australiana de Profesores de Matemáticas e Infancia (2012, p. 1), estas recomendaciones deberían "garantizar que todos los niños tengan acceso a las ideas matemáticas fundamentales y a su aprendizaje durante los primeros años, y al aprendizaje que les prepare para el futuro y favorezca en ellos actitudes positivas". Otra aportación es la declaración conjunta sobre las matemáticas en la Educación Infantil de la Asociación Nacional para la Educación Infantil y el Consejo Nacional de Profesores de Matemáticas de Estados Unidos (NAEYC \& NCTM, 2013). A diferencia de los principios genéricos para la educación matemática del NCTM (2003), en esta declaración se indican algunos aspectos específicos que se deberían considerar en las prácticas matemáticas infantiles. Son recomendaciones que se centran en algunas de las principales necesidades de los niños de las primeras edades para aprender matemáticas:

- Potenciar el interés natural de los niños en las matemáticas y su disposición a utilizarlas para dar sentido a su mundo físico y social.

- Aprovechar las experiencias y conocimientos previos de los niños, incluidos los familiares, lingüísticos, culturales y los de su comunidad, sus aproximaciones individuales al aprendizaje; y sus conocimientos informales.

- Basar los currículos de matemáticas y prácticas docentes en el conocimiento del desarrollo cognitivo, lingüístico, físico, social y emocional de los niños.

- Utilizar currículos y prácticas docentes que fortalezcan los procesos infantiles de resolución de problemas y razonamiento, así como los de representación, comunicación y conexión de ideas matemáticas.

- Asegurar que el currículo sea coherente y compatible con las relaciones y secuencias conocidas de las ideas matemáticas fundamentales.

- Facilitar que los niños interactúen de forma continuada y profunda con las ideas matemáticas clave. 
- Integrar las matemáticas con otras actividades y viceversa.

- Proporcionar tiempo, materiales y apoyo del profesor para que los niños se impliquen en contextos de juego para explorar y manipular ideas matemáticas.

- Introducir activamente conceptos matemáticos, métodos y lenguaje a través de una variedad de experiencias y estrategias de enseñanza apropiadas.

- Apoyar el aprendizaje mediante la evaluación continua y reflexiva del conocimiento, destrezas y estrategias de todos los niños.

Considerando el conjunto de aportaciones genéricas y específicas de la educación matemática infantil, en el apartado siguiente se caracteriza un modelo para el diseño y la gestión de buenas prácticas matemáticas en esta etapa. La finalidad es impulsar la alfabetización matemática de los alumnos de las primeras edades, entendida como "capacidad individual para identificar y comprender el papel que desempeñan las matemáticas en el mundo, emitir juicios bien fundados, utilizar las matemáticas y comprometerse con ellas, y satisfacer las necesidades de la vida personal como ciudadano constructivo, comprometido y reflexivo" (OECD, 2004, p. 3 ).

\section{Hacia un modelo para fomentar la alfabetización matemática en la infancia}

El punto de partida para la caracterización del Modelo de Alfabetización Matemática en la Infancia son las cinco fases planteadas por Alsina (2016) para el diseño y la gestión de actividades matemáticas competenciales. La Figura 1 señala que se trata de una secuencia continua de fases en un flujo circular, cuya finalidad es impulsar la capacidad de los alumnos para usar de forma comprensiva y eficaz las matemáticas que aprenden en la escuela en una variedad de contextos.

Una vez finalizada la actividad competencial, el alumno dispondrá de un nuevo aprendizaje que va a servirle de base para emprender un nuevo ciclo. En esta nueva secuencia se planificarán otros aprendizajes para que, desde lo concreto, el alumno pueda conectar con lo formal interiorizado en una actividad competencial anterior, aumentando de esta forma la comprensión del conocimiento matemático" (Alsina, 2016, p. 16).

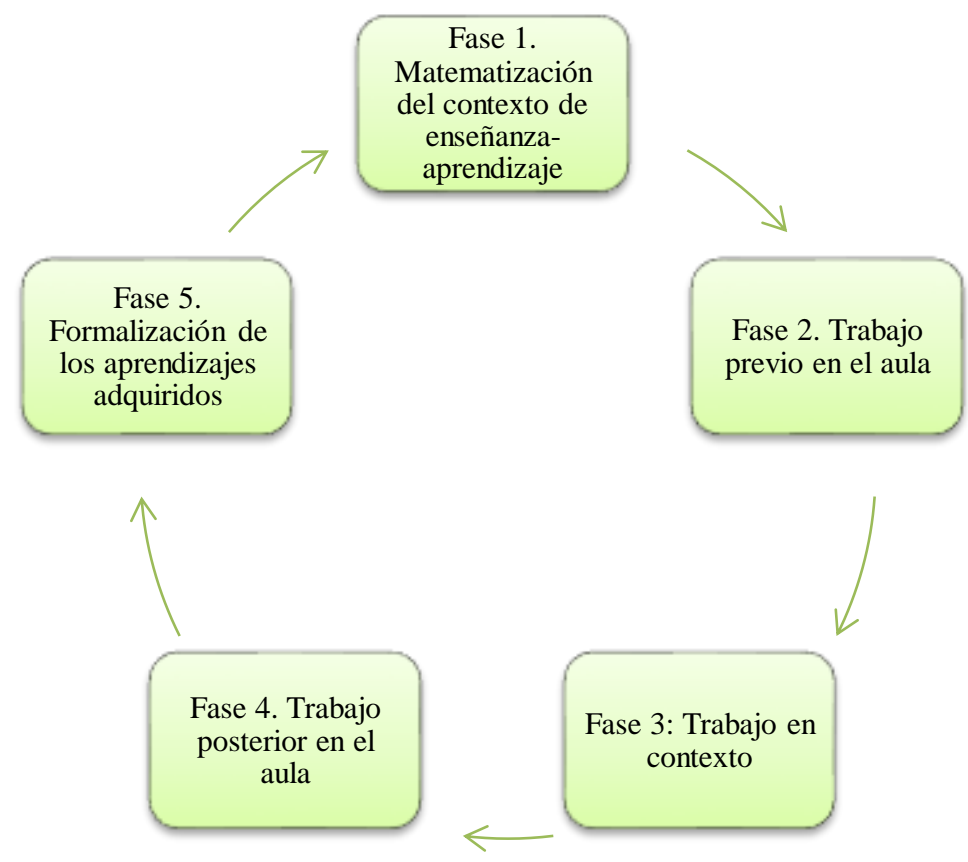

Figura 1. Fases de una actividad matemática competencial (Alsina, 2016) 
El modelo de alfabetización matemática en la infancia contempla seis fases.

\section{Fase 1: Matematización del contexto de enseñanza-aprendizaje}

El planteamiento de la primera fase se fundamenta en contribuciones de la Educación Matemática Realista (Freudenthal, 1973, 1991), del NCTM (2003) y de Alsina (2010a, 2014) en relación tanto a la práctica del profesor como al diseño de la enseñanza y su influencia en el desarrollo de la comprensión. Se consideran también rasgos de las buenas prácticas matemáticas en Educación Infantil que hacen alusión explícita a la importancia de considerar el entorno o bien a la necesidad de tener en cuenta los procesos matemáticos para empoderar el aprendizaje de las matemáticas en las primeras edades. En la declaración conjunta de posición sobre las matemáticas en la Educación Infantil, por ejemplo, se recomienda partir de las experiencias y conocimientos previos de los niños sobre su entorno o bien fomentar los procesos infantiles de resolución de problemas y razonamiento, así como los de representación, comunicación y conexión de ideas matemáticas (NAEYC \& NCTM, 2013). Desde este prisma, se ha caracterizado esta fase con el término "matematización del contexto" para reforzar la idea de que el punto de partida es la selección de un contexto real o realista, con el objeto de poder partir del nivel situacional (Freudenthal, 1991).

Una vez planificado el contexto se deberían analizar los conocimientos matemáticos que se pueden trabajar en dicho contexto. Se toman como base los estándares de contenido del NCTM (2003) y se aboga que, junto con analizar los contenidos matemáticos, es recomendable que el profesorado planifique también a través de qué estándares de procesos matemáticos van a trabajarse dichos contenidos. Alsina (2010a) recomienda partir de estas conexiones entre los conocimientos matemáticos ya desde las primeras edades (Figura 2), dado que cuando los alumnos usan las relaciones existentes entre los contenidos matemáticos, entre los procesos matemáticos y las existentes entre ambos, progresa su conocimiento de la disciplina y crece la habilidad para aplicar conceptos y destrezas con más eficacia en diferentes ámbitos de su vida cotidiana.

\begin{tabular}{|c|c|c|c|c|c|}
\cline { 2 - 6 } \multicolumn{1}{c|}{} & $\begin{array}{c}\text { Resolución } \\
\text { de } \\
\text { problemas }\end{array}$ & $\begin{array}{c}\text { Razonamiento } \\
\text { y prueba }\end{array}$ & Comunicación & Conexiones & Representación \\
\hline $\begin{array}{c}\text { Números y } \\
\text { operaciones }\end{array}$ & Álgebra & & & & \\
\hline Geometría & & & & & \\
\hline Medida & & & & & \\
\hline $\begin{array}{c}\text { Análisis de } \\
\text { datos y } \\
\text { probabilidad }\end{array}$ & & & & & \\
\hline
\end{tabular}

Figura 2. Relación cartesiana entre contenidos y procesos matemáticos

Alsina (2014) plantea ideas clave para el profesorado de Educación Infantil en el diseño y gestión de buenas prácticas que consideren los procesos matemáticos.

Fase 2. Conocimientos matemáticos previos de los alumnos 
Esta fase tiene una inspiración sociocultural ya que se asume que toda actividad formativa debería partir de los conocimientos de los alumnos. Como señaló Vigotsky (1978), si la distancia entre lo que el alumno sabe y lo que se planifica que aprenda es demasiado grande, el aprendizaje difícilmente se produce. En el caso que se produzca, será un aprendizaje desconectado del resto, puesto que no será posible realizar ningún tipo de vínculo. Este planteamiento lo recogen también las principales recomendaciones acerca de las buenas prácticas matemáticas en Educación Infantil, al mencionar la necesidad de aprovechar las experiencias y conocimientos de los niños, como ya se ha mencionado en la fase anterior (NAEYC \& NCTM, 2013).

Como se aprecia, se hace alusión implícita a la necesidad de considerar la zona de desarrollo próximo de los alumnos. La principal herramienta de esta segunda fase tiene también un origen sociocultural: se considera que una vez determinado el contexto de enseñanza-aprendizaje es necesario iniciar un diálogo, es decir, fomentar la interacción con los alumnos para poder identificar sus conocimientos y experiencias previas. La comunicación en el aula de matemáticas en Educación Infantil es, pues, un aspecto relevante que se recoge en la declaración de posición sobre las matemáticas en la primera infancia de la Asociación Australiana de Profesores de Matemáticas e Infancia (2012), al recomendar que el profesorado de las primeras edades reconozca el importante papel que juega el lenguaje en el desarrollo de todo aprendizaje.

Existen diversos recursos posibles para hacer emerger conocimientos en un contexto de comunicación en el aula de matemáticas, aunque uno de los más adecuados son las buenas preguntas. Como señala Mercer (2001), en los procesos de interacción, negociación y diálogo, las buenas preguntas se erigen como un instrumento de mediación idóneo ya que permiten avanzar desde unos primeros niveles de concienciación sobre lo que uno ya sabe o es capaz de hacer hacia niveles superiores. Sullivan y Lilburn (2002) exponen que las buenas preguntas para enseñar matemáticas tienen tres características: i) Requieren comprensión de la tarea, aplicación de conceptos y apropiación de estrategias, así como análisis y síntesis de conceptos implicados, ii) Favorecen que los alumnos sean conscientes de lo que saben y lo que no saben, y muestran al maestro si se entienden bien los conceptos o bien se necesitan ayudas complementarias, iii) Permiten varias respuestas aceptables.

Además de identificar los conocimientos previos a través de buenas preguntas, en el diálogo que se establece durante esta fase se debería pactar el material para realizar la práctica (cinta métrica, libreta para anotar descubrimientos y representar ideas matemáticas) y el material para realizar la documentación (cámara digital).

\section{Fase 3: Aprendizaje de conocimientos matemáticos y documentación en contexto}

Durante esta fase es cuando propiamente se desarrolla la actividad matemática en el contexto real o realista elegido. En el Modelo de Alfabetización Matemática en la Infancia se asume que el acceso a las ideas matemáticas debería producirse en el nivel situacional, es decir, en el contexto de la situación. A medida que los alumnos avanzan en su escolaridad, deben impulsarse otros niveles de comprensión: nivel referencial, mediante esquematización y modelos, descripciones, etc.; nivel general, mediante exploración, reflexión y generalización; $y$, finalmente, nivel formal, mediante procedimientos estándares y notación convencional (Freudenthal, 1991).

La práctica docente del profesorado durante el trabajo en contexto debería favorecer que los alumnos comprendan y usen las ideas matemáticas más importantes. Esta idea aparece también en las principales recomendaciones para llevar a cabo buenas prácticas 
matemáticas en Educación Infantil: en la declaración de posición sobre las matemáticas en la primera infancia de la Asociación Australiana de Profesores de Matemáticas e Infancia (2012), por ejemplo, se insiste en la necesidad de asegurar que las ideas matemáticas con las que interactúan los pequeños sean relevantes, y en la declaración conjunta de posición sobre las matemáticas en la Educación Infantil (NAEYC \& NCTM, 2013) se indica que es importante que el currículo de matemáticas tenga en cuenta las ideas matemáticas fundamentales, entre las que destacan los procesos matemáticos.

Para ello, el maestro debería provocar situaciones que inviten a los alumnos a pensar, argumentar, razonar, descubrir, comprobar, comunicar, conectar, modelar o bien representar ideas matemáticas, considerando de forma explícita los procesos matemáticos expuestos por el NCTM (2003) junto con las subcompetencias matemáticas planteadas por Niss (2002) y la OECD (2004). De este modo, es recomendable que la intervención del profesorado en esta fase sea sobre todo a través de la formulación de buenas preguntas que inviten a los alumnos a interactuar entre ellos e indagar. Fortuny y Rodríguez (2012) añaden que es necesario aprender a mirar con sentido para facilitar la interpretación de las interacciones que se producen.

Otro elemento interesante a considerar durante esta fase es la documentación de las acciones de los alumnos. Esta es, de hecho, una de las principales obsesiones de Malaguzzi (2001) que aquí se asume en su totalidad. Para este autor, la manera más idónea para conocer las capacidades infantiles y desvelar una imagen menos retórica de la infancia es realizar una observación desinteresada de la forma original de aprendizaje de los niños, basada en la escucha y el respeto. Rinaldi $(2001$, p. 5) indica que una variada y amplia documentación (fotografías, videos, transcripciones) hace visible los procesos de aprendizaje y las estrategias utilizadas por cada niño:

Es imposible documentar sin observar e interpretar. Por medio de la documentación, el pensamiento o la interpretación, lo documentado llega a ser tangible y capaz de ser interpretado. Las notas, grabaciones y fotografías representan fragmentos de la memoria. Mientras cada fragmento está saturado con la subjetividad de quien documenta, al mismo tiempo es sujeto a la interpretación de otros, como parte de un proceso colectivo de construcción del aprendizaje. En estos fragmentos se encuentra el pasado y también el futuro (por ejemplo: “¿Qué hubiera pasado si...?”). El resultado es un conocimiento abundante, co-construido y enriquecido por las contribuciones de muchos

En síntesis, la documentación tiene un papel relevante porque sirve al profesorado para llevar a cabo procesos de reflexión acerca de la propia práctica, ayuda a los alumnos a ser conscientes de sus aprendizajes y permite comunicar el trabajo realizado a toda la comunidad educativa y a las familias.

\section{Fase 4. Co-construcción y reconstrucción de conocimiento matemático en el aula}

Esta fase es fundamental para que los alumnos compartan los conocimientos adquiridos en contexto, consiguiendo de esta forma fomentar la co-construcción de nuevo conocimiento matemático a través del andamiaje colectivo, así como la consolidación o reconstrucción de ideas matemáticas adquiridas previamente. De este modo, se considera que el aprendizaje de las matemáticas es una actividad social, tal como se señala en la declaración de posición sobre las matemáticas en la primera infancia de la Asociación Australiana de Profesores de Matemáticas e Infancia (2012).

Los procesos de co-construcción y reconstrucción en un contexto de interacción, negociación y diálogo se han planteado desde el modelo de formación del profesorado realista y reflexivo (Korthagen, 2001; Melief, Tigchelaar, \& Korthagen, 2010; Tigchelaar, Meleif, van Rijswijk, \& Korthagen, 2010). En el ámbito de la educación 
matemática, Alsina (2007, 2010b) y Flores (2007) han realizado trabajos que, desde esta perspectiva, se han focalizado en la formación del profesorado. Algunos estudios también han analizado cómo los alumnos de las primeras edades co-construyen y reconstruyen sus conocimientos matemáticos (Alsina, 2011).

El punto de anclaje de estos trabajos es la interacción, que a su vez es el elemento fundamental de esta fase. Se trata de promover que los alumnos comuniquen lo que han aprendido en contexto, procurando en todo momento que utilicen un lenguaje matemático adecuado, en sintonía también con algunas de las principales recomendaciones sobre buenas prácticas matemáticas en Educación Infantil, que preconizan la importancia del lenguaje para describir, explicar y justificar ideas matemáticas (Asociación Australiana de Profesores de Matemáticas e Infancia, 2012).

Desde los auspicios del modelo sociocultural, Planas (2005) analiza la diversidad de significados surgidos en las interacciones sociales en el aula de matemáticas, los elementos que dificultan la construcción de significados compartidos o bien la carencia de negociación. Los trabajos de esta autora señalan que la enseñanza de las matemáticas en cualquier nivel educativo, incluida la Educación Infantil, requiere integrar procesos de interacción, diálogo y negociación alrededor de los contenidos matemáticos y su gestión, puesto que los alumnos a menudo interpretan las normas establecidas de maneras diferentes y de modo distinto a lo que el profesorado espera. Puig Adam (1955, p. 5), en su decálogo de la educación matemática, escribe:

Es muy difícil definir bien cuando no se domina aún el lenguaje, tanto más difícil cuanto más primario es el concepto definido, como ocurre con gran parte de los conceptos matemáticos. No juzguemos como ignorancia de un concepto o de una propiedad la dificultad de su enunciación. Pese a esta dificultad el niño puede tener clara consciencia de uno y de otra y saberlos aplicar impecablemente. En tales casos, antes de exigir prematuramente repeticiones memorísticas, es preferible esperar a que la perfección expresiva se alcance como consecuencia natural de la fidelidad al pensamiento y del progresivo dominio del lenguaje.

Esta es una de las principales funciones de la comunicación y, en consecuencia, uno de los principales propósitos de esta fase del modelo.

\section{Fase 5. Formalización de los conocimientos matemáticos adquiridos}

Desde los auspicios de la EMR se preconiza que los aprendices pasan por distintos niveles de comprensión del conocimiento matemático, que van desde el nivel situacional (en el contexto de la situación) al nivel formal (conocimientos y notaciones convencionales). Estos niveles implican estrategias, modelos y lenguajes de distinta categoría cognitiva y no constituyen una jerarquía ordenada (Gravemeijer, 1994), pero en definitiva persiguen que progresivamente los alumnos sean capaces de representar la realidad -que es concreta- de forma abstracta (simbólica, formal).

Treffers (1987) indica que se accede al nivel formal a través de la matematización progresiva, bajo dos formas: a) matematización horizontal, que consiste en convertir una situación problemática contextualizada en un problema matemático, basándose en intuición, sentido común, aproximación empírica, observación y experimentación inductiva; b) matematización vertical, que conlleva estrategias de reflexión, generalización, prueba, rigor (limitando interpretaciones y validez), simbolización y esquematización con el objeto de lograr mayores niveles de formalización matemática.

En la etapa de Educación Infantil es necesario, pues, promover que los alumnos empiecen a representar de manera simbólica las situaciones concretas de la realidad 
(Alsina, 2006). Por esta razón, una buena práctica debería finalizar, a medida que avanzan las posibilidades de representación de los alumnos, con la formalización de los aprendizajes matemáticos adquiridos. Los alumnos deberían ir adquiriendo progresivamente herramientas que les permitan formalizar los aprendizajes a través del lenguaje escrito en general y del lenguaje algebraico en particular. La Figura 3 muestra un ejemplo de abstracción progresiva de la idea matemática "dos".

\begin{tabular}{|c|c|c|}
\hline & $\mathbf{X}$ \\
\hline $\begin{array}{l}\text { Representación concreta de } \\
\text { "dos" en contexto (dibujo de } \\
\text { dos huevos rotos y asociación } \\
\text { término a término) }\end{array}$ & $\begin{array}{l}\text { Representaciones pictóricas } \\
\text { de "dos" (signos y asociación } \\
\text { término a término) }\end{array}$ & $\begin{array}{l}\text { Representación abstracta } \\
\text { de "dos" (símbolo propio } \\
\text { del lenguaje matemático) }\end{array}$ \\
\hline
\end{tabular}

Figura 3. Abstracción progresiva de la idea matemática "dos"

En las primeras edades no es aconsejable asociar el aprendizaje de las matemáticas exclusivamente a lo formal. Se insiste en esta idea puesto que, tradicionalmente, se ha considerado la adquisición de conocimiento formal de forma prematura. Así, en la enseñanza de los primeros números naturales, una práctica muy habitual ha consistido en vincular su aprendizaje exclusivamente a la adquisición de la notación convencional. La Figura 4 muestra un ejemplo de práctica descontextualizada de la idea matemática "dos" propia del enfoque de destrezas (Baroody \& Coslick, 1998):

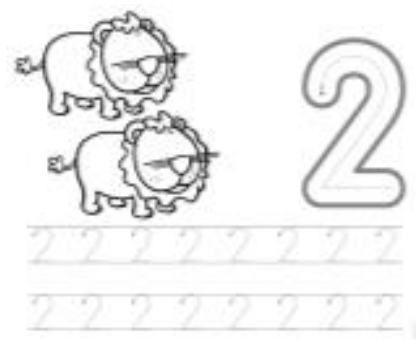

Figura 4. Práctica descontextualizada de la idea matemática "dos"

En el Modelo de Alfabetización Matemática en la Infancia, la práctica anterior tiene sentido sólo si previamente se ha accedido al conocimiento en situaciones contextualizadas. Por esta razón, la fase de formalización se sitúa en las fases finales de la secuencia o itinerario didáctico.

Fase 6: Reflexión sistemática sobre la práctica matemática realizada

Para cerrar la secuencia de fases es imprescindible contemplar la reflexión sistemática a partir de la propia acción, con el objeto de mejorarla, tal como sugieren las principales recomendaciones sobre buenas prácticas matemáticas en Educación Infantil. En la declaración de posición sobre las matemáticas en la primera infancia de la Asociación Australiana de Profesores de Matemáticas e Infancia (2012), por ejemplo, se indica que la evaluación debería usarse para planificar nuevas actividades e intervenciones, y en la declaración conjunta de posición sobre las matemáticas en la Educación Infantil (NAEYC \& NCTM, 2013) se hace alusión explícita a la relevancia de apoyar el aprendizaje mediante la evaluación continua y reflexiva. 
En el contexto de la formación del profesorado inicial y permanente, la reflexión sistemática sobre la propia práctica ha generado mucha literatura. Schön (1983) planteó que un profesional debe saber, saber hacer, saber moralmente bien y reflexionar sobre su acción. Para este autor, pues, el maestro necesita de la reflexión planificadora antes de emprender una acción formativa; de la reflexión activa o aquella que se ejecuta en la práctica real y de la post-activa que evalúa la práctica finalizada. En todos los casos, la reflexión tiene que ser una reflexión documentada, contrastada y que permita poner en marcha procesos de reajuste y mejora. Posteriormente, otros autores han trabajado alrededor de la competencia reflexiva del profesorado para mejorar su práctica (Korthagen, 2001; Melief, Ticgchelaar \& Korthagen, 2010; Perrenaud, 2004; Tigchelaar, Melief, van Rijswijk \& Korthagen, 2010).

Arcavi (2016) desarrolla el proyecto VIDEO-LM para indagar cómo apoyar a los profesores hacia la toma de conciencia acerca de su propia práctica profesional y la consecuente toma idónea de decisiones en el aula. VIDEO-LM se inspira en el estudio de clases japonés (Lesson Study) y los principios esenciales del marco de Schoenfeld (2010), que sostiene y demuestra que es posible describir, explicar y hasta predecir la toma de decisiones del profesor y sus acciones subsiguientes en términos de sus conocimientos, sus objetivos y sus creencias/predisposiciones. Con este proyecto, pues, se pretende desarrollar la capacidad de reflexión acerca de las prácticas de aula en base a observaciones y análisis de videos de clase. Para lograr este propósito, se contemplan seis componentes de reflexión para mejorar la propia práctica: 1) ideas matemáticas y meta-matemáticas de la lección; 2) objetivos explícitos e implícitos atribuibles al docente; 3) tareas asignadas y su desarrollo en la clase; 4) interacciones profesoralumno; 5) dilemas docentes durante la clase y su resolución; 6) creencias del profesor acerca de las matemáticas, su enseñanza y su aprendizaje inferidos de la lección.

Llevar a cabo una reflexión sistemática con el objeto de mejorar la propia práctica implica preguntarse acerca de los conocimientos matemáticos, que hacen referencia al listado de contenidos y procesos que deberían explicitarse en la fase 1 de nuestro modelo (¿qué se enseña); los conocimientos didácticos, que hacen referencia a la planificación y la gestión de la práctica docente descritos en las fases 2 a 5 de nuestro modelo (¿cómo se enseña?); y lo que piensa el profesor acerca de lo que enseña y cómo lo enseña, que se incluye en esta fase final del modelo (¿qué se puede mejorar?).

Algunos autores han elaborado instrumentos que pueden ayudar al profesorado de las primeras edades a analizar y reflexionar acerca de sus prácticas matemáticas. Coronata (2014) y Alsina y Coronata (2014) elaboran un instrumento para analizar la presencia de procesos matemáticos en la práctica docente. El diseño, construcción y validación del instrumento sigue seis fases: 1) análisis histórico-epistemológico de procesos matemáticos y sus significados; 2) estudio de investigaciones sobre procesos matemáticos en las prácticas docentes del profesorado de Educación Infantil; 3) análisis del tratamiento otorgado a los procesos matemáticos en el currículo; 4) construcción de la versión piloto del instrumento; 5) revisión mediante juicio de expertos; y 6) construcción de la versión final del instrumento. Maurandi, Alsina y Coronata (en prensa) han analizado la fiabilidad del instrumento a partir de 95 entrevistas a maestros. Los datos se han analizado con el paquete estadístico $R$ Core Team (R versión 3.1.0) sobre una plataforma i686-pc-linux-gnu (32-bit). Con el paquete $P$ sych se ha calculado el coeficiente alfa de Cronbach: resolución de problemas (0.86); razonamiento y prueba (0.88); conexiones (0.82); comunicación (0.81) y representación (0.79). Esto permiten afirmar que todos los ítems están relacionados significativamente con aquellos construidos para evaluar la misma faceta del factor, por lo que forman parte del mismo 
constructo (Revelle, 2015). Este instrumento constituye una herramienta base para medir la presencia o ausencia de procesos matemáticos en la práctica docente, por lo que puede ser útil para apoyar y guiar la reflexión sistemática sobre la práctica matemática.

\section{Consideraciones finales}

Se ha presentado la caracterización de un modelo para fomentar la alfabetización matemática en la infancia que, por sus características, puede ser aplicado en los distintos niveles escolares de Educación Infantil, y que podría ser extrapolable a la etapa de Educación Primaria. Para el diseño del modelo se han considerado contribuciones que provienen tanto de la investigación en educación matemática como de la investigación en educación matemática infantil en particular. En concreto, el modelo se fundamenta principalmente en las aportaciones del $\operatorname{NCTM}(2003,2015)$, las declaraciones de posición sobre la educación matemática infantil de varios organismos internacionales (Asociación Australiana de Profesores de Matemáticas e Infancia, 2012; NAEYC \& NCTM, 2013), así como los principios de la EMR (Freudenthal, 1973, 1991). El diagrama siguiente esquematiza las seis fases que de momento tiene el modelo descrito para fomentar la alfabetización matemática en la infancia:

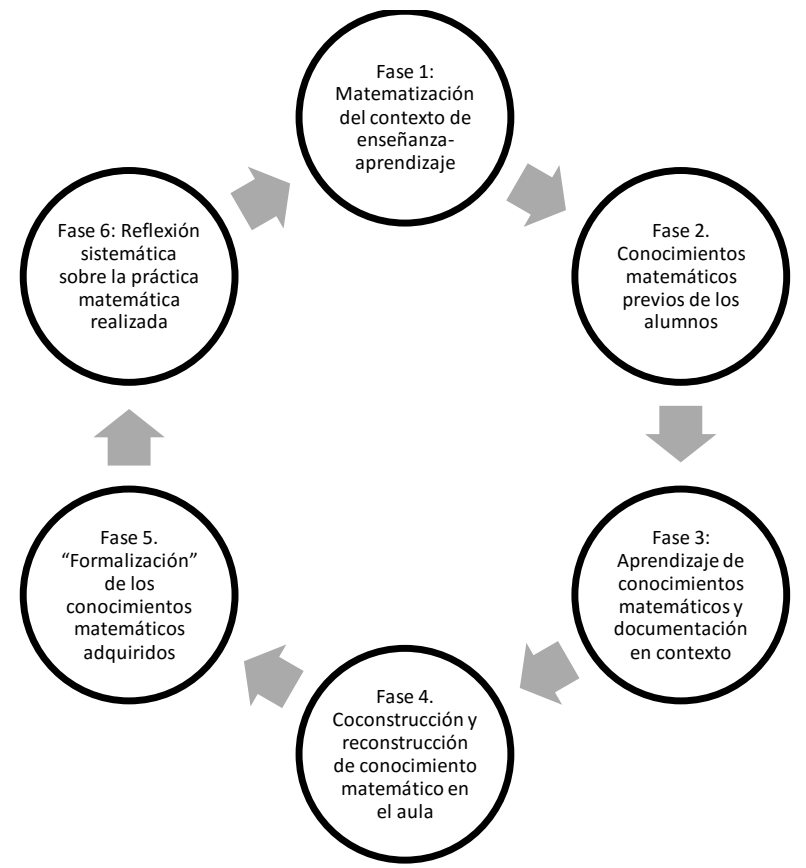

Figura 5. Modelo de Alfabetización Matemática en la Infancia

Como en la secuencia planteada en Alsina (2016) para el diseño y gestión de actividades matemáticas competenciales, el modelo aquí presentado parte de que, una vez finalizada la práctica docente en el aula, el alumno debería haber aprendido un nuevo conocimiento matemático importante con profundidad y comprensión que, a su vez, ha de ser punto de partida de un nuevo aprendizaje. El maestro, a partir de la reflexión sistemática de su práctica, planificará nuevas prácticas para que, desde lo concreto, el alumno pueda conectar nuevos aprendizajes con el conocimiento (formal) interiorizado en una práctica matemática anterior. Todo ello, para fomentar el uso comprensivo y eficaz del conocimiento matemático, es decir, la alfabetización matemática del alumno.

Uno de los principales desafíos al presentar un modelo de este tipo consiste en producir "indicadores de éxito", que a su vez implica definir "éxito" en un modelo de este tipo (Arcavi, 2016). Consideramos que los indicadores del instrumento para analizar la presencia de procesos matemáticos en la práctica docente (Coronata, 2014; Alsina \& 
Coronata, 2014) pueden ser el punto de partida para obtener datos en el sentido planteado por Arcavi. En el futuro será necesario diseñar nuevos estudios orientados, por lo menos, en una triple dirección: 1) establecer nuevos indicadores para analizar la eficacia del Modelo de Alfabetización Matemática en la Infancia; 2) determinar los principales beneficios y las dificultades que plantea el modelo descrito; 3) replantear, suprimir o incorporar nuevas fases con el propósito de que finalmente sea un modelo útil para diseñar, gestionar y evaluar buenas prácticas matemáticas en el aula de Educación Infantil que fomenten la alfabetización matemática de los alumnos.

\section{Referencias}

Alsina, Á. (2006). Cómo desarrollar el pensamiento matemático de 0 a 6 años. Barcelona: Editorial Octaedro-Eumo.

Alsina, Á. (2007). El aprendizaje reflexivo en la formación permanente del profesorado: un análisis desde la didáctica de la matemática. Educación Matemática, 19(1), 99126.

Alsina, Á. (2010a). La "pirámide de la educación matemática", una herramienta para ayudar a desarrollar la competencia matemática. Aula de Innovación Educativa, 189, 12-16.

Alsina, Á. (2010b). El aprendizaje reflexivo en la formación inicial del profesorado: un modelo para aprender a enseñar matemáticas. Educación Matemática, 22(1), 149166.

Alsina, Á. (2011). Educación matemática en contexto de 3 a 6 años. Barcelona: ICEHorsori.

Alsina, Á. (2013). Educación Matemática en Infantil: Investigación, Currículum, y Práctica Educativa. REDIMAT, 2(1), 100-153.

Alsina, Á. (2014). Procesos matemáticos en Educación Infantil: 50 ideas clave. Números $86,5-28$.

Alsina, Á. (2016). Diseño, gestión y evaluación de actividades matemáticas competenciales en el aula. Épsilon, 33(1), 7-29.

Alsina, Á., \& Coronota, C. (2014). Los procesos matemáticos en las prácticas docentes: diseño, construcción y validación de un instrumento de evaluación. Edma 0-6: Educación Matemática en la Infancia, 3(2), 21-34.

Arcavi, A. (2016). Promoviendo conversaciones entre docentes acerca de clases filmadas de Matemáticas. Cuadernos de Investigación y Formación en Educación Matemática, 15, 385-396.

Asociación Australiana de Profesores de Matemáticas e Infancia (2012). Declaración de posición sobre las matemáticas en la primera infancia. Edma 0-6: Educación Matemática en la Infancia, 1(2), 1-4.

Baroody, A. J., \& Coslick, R.T. (1998). Fostering Children's Mathematical Power: An Investigative Approach to K-8 Mathematics Instruction. Mahwah: Lawrence Erlbaum Associates.

Clements, D. H. (2004). Major themes and recommendations. En D.H. Clements, J. Sarama, \& A.M. DiBiase (Eds.), Engaging young children in mathematics: Standards for early childhood mathematics education (pp. 7-72). Mahwah: Lawrence Erlbaum Associates. 
Clements, D. H., \& Sarama, J. (2009). Learning and teaching early math: The learning trajectories approach. Nueva York: Routledge.

Coronata, C. (2014). Presencia de los procesos matemáticos en la enseñanza del número de 4 a 8 años. Transición entre la Educación Infantil y Primaria. Trabajo de Tesis Doctoral. Universidad de Girona.

de Castro, C. (2007). La evaluación de métodos para la enseñanza y el aprendizaje de las matemáticas en la Educación Infantil. UNION, 11, 59-77.

de Castro, C. (2016). El estudio de documentos curriculares como organizador de la investigación en educación matemática infantil. En J. A. Macías y otros (Eds.), Investigación en Educación Matemática XX (pp. 39-52). Málaga: SEIEM.

de Castro, C., Flecha, G., \& Ramírez, M. (2015). Matemáticas con dos años: buscando teorías para interpretar la actividad infantil y las prácticas docentes. Tendencias Pedagógicas, 26, 89-108.

Edo, M. (2016). Emergencia de la Investigación en Educación Matemática Infantil. Juego y Matemáticas. En J. A. Macías y otros (Eds.), Investigación en Educación Matemática XX (pp. 53-66). Málaga: SEIEM.

Flores, P. (2007). Profesores de matemáticas reflexivos: formación y cuestiones de investigación. PNA, 4(1), 139-159.

Fortuny, J. M., \& Rodríguez, R. (2012). Aprender a mirar con sentido: facilitar la interpretación de las interacciones en el aula. Avances de Investigación en Educación Matemática, 1, 23-37.

Freudenthal, H. (1973). Mathematics as an Educational Task. Dordrecht: Reidel Publishing Company.

Freudenthal, H. (1991). Revisiting mathematics education. Dordrecht: Kluwer.

Fuson, K. C., Clements, D. H., \& Beckman, S. (2009). Focus in prekindergarten: Teaching with curriculum focal points. Reston: NCTM\& NAEYC.

Gómez, P., Cañadas, M. C., Bracho, R., Restrepo, A. M., \& Aristizábal, G. (2011). Análisis temático de la investigación en Educación Matemática en España a través de los Simposios de la SEIEM. En M. Marín, G. Fernández, L. J. Blanco \& M. Palarea (Eds.), Investigación en Educación Matemática XV (pp. 371-382). Ciudad Real: SEIEM.

Gravemeijer, K.P.E. (1994). Developing realistic mathematics education. Utrecht: CDß Press Freudenthal Institute.

Korthagen, F. A. J. (2001), Linking practice and theory. The pedagogy of realistic teacher education. Londres: Lawrence Erlbaum Associates.

López, M. \& Alsina, Á. (2015). La influencia del método de enseñanza en la adquisición de conocimientos matemáticos en educación infantil. Edma 0-6: Educación Matemática en la Infancia, 4(1), 25-34.

Malaguzzi, M. (2001). La educación infantil en Reggio Emilia. Barcelona: Rosa SensatOctaedro.

Maurandi, A., Alsina, Á., \& Coronata, C. (en prensa). Los procesos matemáticos en la práctica docente: análisis de la fiabilidad de un cuestionario de evaluación. Educatio S. XX1. 
Melief, K., Tigchelaar, A., \& Korthagen, F. (2010). Aprender de la práctica. En O. Esteve, K. Melief \& Á. Alsina (Eds.), Creando mi profesión. Una propuesta para el desarrollo profesional del profesorado (pp. 19-38). Barcelona: Editorial Octaedro.

Mercer, N. (2001). Palabras y mentes. Barcelona: Paidós.

NAEYC \& NCTM (2013). Matemáticas en la Educación Infantil: Facilitando un buen inicio. Declaración conjunta de posición. Edma 0-6: Educación Matemática en la Infancia, 2(1), 1-23.

NCTM (2003). Principios y estándares para la educación matemática. Sevilla: Thales.

NCTM (2015). De los principios a la acción. Para garantizar el éxito matemático de todos. Reston: NCTM.

Niss, M. (2002). Mathematical competencies and the learning of mathematics: the Danish Kom Project. Roskilde: Roskilde University.

NRC (2014). Fundamentos cognitivos para la iniciación en el aprendizaje de las matemáticas. Edma 0-6: Educación Matemática en la Infancia, 3(1), 21-48.

OECD (2004). Learning for tomorrow's world: First results from PISA 2003. París: OECD.

OECD (2007). PISA 2006 Science competence for tomorrow's world. París: OECD.

Perrenoud, P. (2004). Desarrollar la práctica reflexiva en el oficio de enseñar. Profesionalización y razón pedagógica. Barcelona: Graó.

Planas, N. (2005). El papel del discurso en la construcción del Discurso de la práctica matemática. Cultura y Educación, 17(1), 19-34.

Planas, N. \& Alsina, A. (2014). Epílogo. En N. Planas y A. Alsina (Eds.), Educación matemática y buenas prácticas (pp. 265-272). Barcelona: Graó (2ª edición).

Price, M. H. (1986). The Perry Movement in school mathematics. En M. H. Price (Ed.), The development of the secondary curriculum (pp. 7-27). Londres: Croom Helm.

Puig Adam, P. (1955). Decálogo de la didáctica de la matemática media. La Gaceta Matemática, $1^{a}$ Serie, Tomo VII, 5 y 6, 2-6.

Revelle, W. (2015). Psych: procedures for personality and psychological research. Evanston: Northwestern University.

Rinaldi, C. (2001) The pedagogy of listening: The listening perspective from Reggio Emilia. Children in Europe, 1, 1-5.

Schoenfeld, A.H. (2010). How we think: a theory of goal-oriented decision making and its educational applications. Nueva York: Routledge.

Schön, D. (1983). The reflective practitioner. How professionals think in action. Londres: Temple Smith.

Sierra, T. A., \& Gascón, J. (2011). Investigación en Didáctica de las Matemáticas en la Educación Infantil y Primaria. En M. Marín, G. Fernández, L. J. Blanco \& M. Palarea (Eds.), Investigación en Educación Matemática XV (pp. 125-163). Ciudad Real: SEIEM.

Sullivan, P., \& Lilburn, P. (2002). Good questions for math teaching. Sydney: Oxford University Press. 
Tigchelaar, A., Melief, K., van Rijswijk, M., \& Korthagen, F. (2010). Elementos de una posible estructura del aprendizaje realista en la formación inicial y permanente delprofesorado. En O. Esteve, K. Melief \& A. Alsina (Eds.), Creando mi profesión. Una propuesta para el desarrollo profesional del profesorado (pp. 3964). Barcelona: Editorial Octaedro.

Treffers, A. (1987). Three dimensions. A model of goal and theory description in mathematics instruction. The Wiskobas Project. Dordrecht: Reidel Publishing Co.

Vigotsky, L.S. (1978) Mind in society. The development of higher psychological processes. Cambridge: Harvard University Press.

\section{Referencia del autor}

Ángel Alsina, Universidad de Girona (España). angel.alsina@udg.edu 


\section{Characterization of a mathematical model to empower mathematical literacy in children: linking research with good practices}

In recent decades, various international organizations have indicated the benefits of mathematics childhood education for both children and society (NCTM, 2003; OECD, 2007). Greater consideration of children's mathematics education has led to: a) the extension of the age range, including children from 0 to 3 years (e.g. Clements \& Sarama, 2009; NRC, 2014); b) the gradual increase of research on childhood mathematics education in the Spanish and European contexts (e.g. Alsina, 2013, Edo, 2016). The purpose of this article is to begin to characterize a model to empower mathematical literacy in childhood, considering the progress in areas and research agendas of childhood mathematics education. The Mathematical Model Childhood Literacy draws on findings about mathematics teaching and good practice. These findings come from research in mathematics education in general and in early childhood mathematics education. Specifically, the model is based on the contributions of NCTM (2003, 2015), position statements on mathematics education child of international organizations (e.g. NAEYC \& NCTM, 2013), and the EMR principles (Freudenthal, 1973, 1991). It is a model of six phases: mathematisation of the teaching and learning context; prior mathematical knowledge of students; learning mathematical knowledge and documentation in context; co-construction and reconstruction of mathematical knowledge in the classroom; formalization of mathematical knowledge; and systematic reflection on the mathematical practice done. It is a continuous sequence of phases in a circular flow: contents and processes for teaching in a real or realistic context (what is taught); teaching knowledge, about planning and management of teaching practice (how do you teach?); and what the teacher thinks about what to teach and how (what can be better?). In short, the model assumes that teaching must lead the student to learn important new mathematical knowledge with depth and understanding. This new knowledge will be the starting point for further learning. It will promote understanding and effective use of mathematical knowledge from the early years. 